\title{
Adrenal function is related to prognosis in moderate community-acquired pneumonia
}

\author{
M. Kolditz, M. Halank, B. Schulte-Hubbert and G. Höffken
}

ABSTRACT: The aim of our study was to prospectively examine adrenal function, including cosyntropin stimulation, and its prognostic value in patients with moderate community-acquired pneumonia (CAP).

59 consecutive adult patients hospitalised on normal wards because of CAP were enrolled. A cosyntropin stimulation test was performed and serum concentrations of C-reactive protein, procalcitonin, interleukin-6, tumour necrosis factor- $\alpha, \mathrm{ACTH}$, cortisol, dehydroepiandrosterone (DHEA) and dehydroepiandrosterone-sulfate (DHEAS) were measured. Predefined outcome parameters were clinical instability after $72 \mathrm{~h}$, mortality and combined intensive care unit (ICU) admission or mortality.

Critical illness-related corticosteroid insufficiency (CIRCI) occurred in six patients (10.3\%). Cortisol, age-corrected DHEA, ACTH and the DHEA/DHEAS ratio were elevated in patients remaining unstable after $72 \mathrm{~h}$. In multivariate analysis, cortisol $(p=0.03)$, ACTH $(p=0.04)$ and the pneumonia severity index $(\mathrm{PSI})$ score $(\mathrm{p}=0.005)$ independently predicted clinical instability after $72 \mathrm{~h}$, and only cortisol predicted mortality $(\mathrm{p}=0.04)$ and combined ICU-admission or mortality $(p=0.006)$. The predictive value of serum cortisol after receiver operating characteristic curve analysis equalled that of the PSI score. Patients with serum cortisol $>734 \mathrm{nmol} \cdot \mathrm{L}^{-1}$ had a high probability for mortality (OR $38.3 ; p=0.002)$.

$\mathrm{CIRCI}$ is rare in patients with moderate CAP. Adrenal function is related to the prognosis of CAP. The diagnostic accuracy of serum cortisol equals that of the PSI score. Serum cortisol should be evaluated within clinical prediction scores on larger studies.

KEYWORDS: Adrenal function, community-acquired pneumonia, cortisol, prognosis, risk prediction

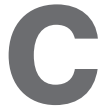
oncentration changes of adrenal hormones such as cortisol, dehydroepiandrosterone (DHEA) and DHEA sulfate (DHEAS) have been described in patients with severe sepsis and septic shock and linked to the prognosis of the disease [1, 2]. Additionally, changes in the ratios of these hormones (DHEA/ DHEAS and cortisol/DHEA) might be associated with the development of septic shock and sepsis outcome, suggesting the importance of a balanced adrenal response to maintain an adequate inflammatory response to acute illness [1, 2].

Critical illness-related corticosteroid insufficiency (CIRCI) is considered as an inadequate adrenal response to critical illness-related stress together with tissue corticosteroid resistance, and is characterised by an exaggerated pro-inflammatory response [3]. A recent consensus conference suggested, that the diagnosis is best made by a change in total serum cortisol of $<248 \mathrm{nmol} \cdot \mathrm{L}^{-1}$ after cosyntropin stimulation [3]. CIRCI was repeatedly associated with unfavourable outcome in patients with septic shock [3-5], even though therapeutic trials with low-dose hydrocortisone showed conflicting results [6].

Community-acquired pneumonia (CAP) is one of the predominant causes and precursors of sepsis and septic shock. Preliminary data from a small study in CAP patients suggested a beneficial effect of low-dose hydrocortisone infusion even in the absence of septic shock, although the underlying mechanism for this effect has not been evaluated and adrenal function was not measured in that study [7].

In contrast to the possibility of CIRCI contributing to this postulated treatment effect in CAP, two recent studies found higher levels of serum cortisol to be correlated with CAP severity and associated with nonsurvival in CAP, with a predictive accuracy comparable to that of the pneumonia severity index (PSI) score [8, 9]. However, in the study of CHRIST-CRAIN et al. [8], cosyntropin stimulation had not been performed to elucidate adrenal dysfunction. In the study of GOTOH et al. [9], CIRCI was confounded by the

\section{AFFILIATIONS}

Dept of Pulmonology, Medical Clinic 1, University Hospital Carl Gustav Carus, Dresden, Germany.

CORRESPONDENCE

M. Kolditz

Dept of Pulmonology, Medical Clinic 1

University Hospital Carl Gustav Carus Fetscherstr. 74

01307 Dresden Germany

E-mail: martin.kolditz@ uniklinikum-dresden.de

Received:

Dec 022009

Accepted after revision: Jan 112010 First published online: Jan 282010 
inclusion of patients with septic shock; nevertheless, it was not found to be a useful prognostic tool in the collective. Neither study examined DHEA and DHEAS.

The aim of our study was to prospectively examine adrenal function including cortisol, adrenocorticotropic hormone (ACTH), DHEA and DHEAS concentrations before and after cosyntropin stimulation, and their prognostic accuracy in comparison to established inflammatory parameters to predict the outcome of patients with moderate CAP. In this way, we aimed to elucidate possible diagnostic properties of these parameters as well as possible explanations of the postulated therapeutic effects of steroids in CAP without septic shock.

\section{METHODS}

59 consecutive adult patients, hospitalised between May 2007 and March 2009 for $\geqslant 24 \mathrm{~h}$, at the emergency department or the normal ward, because of CAP, were prospectively enrolled in the study. CAP was defined as presence of a new infiltrate on chest radiography and at least one of the following: fever $\left(\geqslant 38.3^{\circ} \mathrm{C}\right)$, cough, presence of purulent sputum or focal chest signs on auscultation. Patients were excluded from the study if one of the following was present: nosocomial pneumonia, initial admission to the intensive care unit (ICU), immunosuppression (including HIV or recent chemotherapy), active tuberculosis, sarcoidosis, pregnancy, concomitant medication with steroids (either acute or chronic including inhaled steroids), medication with ketoconazole or contraceptive drugs, known allergy against tetracosactid, or history of adrenal, hypothalamic or pituitary disease.

The study protocol was approved by the local ethics committee. Informed consent was obtained from the participants. Disease severity was calculated according to the CRB-65 (confusion, respiratory rate $\geqslant 30$ breaths $\cdot \mathrm{min}^{-1}$, low blood pressure (systolic value $<90 \mathrm{mmHg}$ or diastolic value $\leqslant 60 \mathrm{mmHg}$ ) and age $\geqslant 65 \mathrm{yrs}$ ), CURB (confusion, urea $>7 \mathrm{mmol} \cdot \mathrm{L}^{-1}$, respiratory rate $\geqslant 30$ breaths $\cdot \mathrm{min}^{-1}$ and low blood pressure) and PSI scores.

On the morning after hospital admission (day 1), a cosyntropin stimulation test was performed with intravenous injection of $0.25 \mathrm{mg}$ tetracosactrin (Synacthen ${ }^{\circledR}$, Novartis, Basel, Switzerland) and collection of blood samples immediately before the test and 30 and $60 \mathrm{~min}$ after injection. Concentrations of the following parameters were measured from the first blood sample directly after collection: C-reactive protein (CRP) (particle-enhanced immunoturbidimetric assay; Roche Diagnostics $\mathrm{GmbH}$, Mannheim, Germany; normal range $<5 \mathrm{mg} \cdot \mathrm{L}^{-1}$ ), procalcitonin (PCT) (enzyme-labelled sequential chemiluminescent immunoassay (LIA); Brahms AG, Germany; normal range $<0.5 \mathrm{ng} \cdot \mathrm{mL}^{-1}$ ), interleukin (IL)-6 (LIA; Siemens Medical Solutions Diagnostics, Los Angeles, CA, USA, normal range $<2-3,5 \mathrm{pg} \cdot \mathrm{mL}^{-1}$ ), tumour necrosis factor (TNF)- $\alpha$ (enzymelabelled sequential LIA, Siemens Medical Solutions Diagnostics; normal range $<8.1 \mathrm{pg} \cdot \mathrm{mL}^{-1}$ ), ACTH (two-site sequential LIA; Siemens Medical Solutions Diagnostics; normal range 2.6$10.1 \mathrm{pmol} \cdot \mathrm{l}^{-1}$ ), and cortisol (antigen-linked technique LIA; DiaSorin, Saluggia, Italy; normal range $119-618 \mathrm{nmol} \cdot \mathrm{L}^{-1}$ ). Afterwards, aliquots of serum were stored frozen at $-20^{\circ} \mathrm{C}$, and the following parameters were analysed $\leqslant 2$ weeks after collection: DHEA (competitive coated tube radioimmunoassay;
Diagnostic Systems Laboratories Inc., Webster, TX, USA) and DHEAS (competitive LIA; Siemens Medical Solutions Diagnostics). As normal ranges of these hormones are age(and sex-) dependent, the percentage of age- (and, for DHEAS, sex-) matched normal values was calculated as previously described [2, 10]. In the blood samples collected after tetracosactrin injection, cortisol, DHEA, DHEAS and ACTH were measured.

CIRCI was defined as a maximum difference in cortisol concentration of $<248 \mathrm{nmol} \cdot \mathrm{L}^{-1}$ [3]. Basal cortisol levels were not included in the definition, as we felt that basal concentrations derived from septic shock patients were not appropriate for the less severely ill patients of our cohort.

Antibiotics were administered for empirical therapy as recommended by the German guidelines for CAP [11].

Predefined outcome parameters were hospital and 30-day mortality, combined hospital mortality and ICU admission, and clinical instability on day 4 (72 h after the cosyntropin stimulation test). Clinical instability was defined as absence of one of the following criteria defining clinical stability: heart rate $\leqslant 100$ beats $\cdot \mathrm{min}^{-1}$, respiratory rate $\leqslant 24$ breaths $\cdot \mathrm{min}^{-1}$, systolic blood pressure $\geqslant 90 \mathrm{mmHg}$, temperature $\leqslant 37.8^{\circ} \mathrm{C}$, ability to eat, oxygen saturation $\geqslant 90 \%$ and return to baseline mental status [12].

Data from biochemical analyses are presented as median (range). Medians were compared using the nonparametric Mann-Whitney test. Receiver operating characteristic (ROC) curve analysis was used to determine the diagnostic properties of predictive parameters, optimal cut-off values were determined by Youden's index. Statistical comparisons between the areas under the curve (AUC) were calculated according to the Hanley and McNeil method [13] using MedCalc statistical software (MedCalc Software bvba, Mariakerke, Belgium). Odds ratios were calculated by using the Mantel-Haenszel estimate. Univariate and multivariate analyses were performed to predict the binary end points by including the PSI, CRB-65 and CURB scores and all measured serum parameters, for multivariate analyses a logistic regression model with stepwise forward selection was employed. To assess the influence of cortisol concentrations on mortality, we produced a KaplanMeier survival curve comparison. Between the groups was performed by log-rank test. A p-value of $<0.05$ (two-sided) was considered statistically significant. Statistical analyses were performed with SPSS version 15.0 software (SPSS Inc., Chicago, IL, USA).

\section{RESULTS}

One patient was excluded from the analysis because of a final diagnosis of lung cancer instead of pneumonia. The baseline data of the other 58 patients are shown in table 1. In $21(26 \%)$ patients, a microbiological diagnosis could be established, with Streptococcus pneumoniae being the predominant pathogen $(38 \%)$, followed by Mycoplasma pneumoniae, Staphylococcus aureus and Escherichia coli (14\% each). Initial empirical antimicrobial therapy consisted of fluoroquinolone monotherapy in $23(40 \%)$ patients, $\beta$-lactam monotherapy in $19(33 \%)$, and a $\beta$-lactam combined with either a macrolide in nine $(16 \%)$, a fluoroquinolone in six $(10 \%)$ or clindamycin in one $(2 \%)$. 
Six $(10 \%)$ patients died in the hospital. There were no further deaths within the 30 -day follow-up period. Three $(5 \%)$ additional patients were admitted to the ICU after inclusion into the study because of deterioration in their clinical condition; these patients survived. 31 patients (53\%) fulfilled all clinical stability criteria on day 4.

CIRCI occurred in six $(10.3 \%)$ patients, including one nonsurvivor. A trend was noted that more patients with CIRCI were clinically unstable after $72 \mathrm{~h}$ (five out of six patients; OR 7.1 (95\% CI 0.78-65.7); $\mathrm{p}=0,082)$.

As expected, the baseline cortisol level was positively correlated with ACTH (Spearman coefficient of rank correlation $\mathrm{r}=0.423 ; \mathrm{p}=0.001)$. Moreover, cortisol levels were positively correlated with pneumonia severity as defined by the PSI score $(\mathrm{r}=0,414 ; \mathrm{p}=0.001)$ and with all measured inflammatory serum parameters, with the strongest association to IL-6 ( $\mathrm{r}=0.523 ; \mathrm{p}<0.001)$.

\section{TABLE 1 Characteristics of the study patients}

\begin{tabular}{|c|c|c|c|}
\hline & All patients & Survivors & Nonsurvivors \\
\hline Subjects $\mathrm{n}$ & 58 & 52 & 6 \\
\hline Males & $30(52)$ & $29(56)$ & $1(17)$ \\
\hline Age yrs & 72 (20-93) & $71(20-88)$ & 79 (43-93) \\
\hline Comorbidities & $43(74)$ & $37(71)$ & $6(100)$ \\
\hline COPD & $5(9)$ & $4(8)$ & $1(17)$ \\
\hline Smoking history & $21(36)$ & $20(38)$ & $1(17)$ \\
\hline Antibiotic pre-treatment & $12(21)$ & $10(19)$ & $2(33)$ \\
\hline Pathogen established & $21(36)$ & $20(38)$ & $1(17)$ \\
\hline Bacteraemia & $6(10)$ & $6(12)$ & $0(0)$ \\
\hline $\begin{array}{l}\text { Length of hospital stay } \\
\text { days }\end{array}$ & $8(2-72)$ & $8(2-72)$ & $2.5(2-6)^{*}$ \\
\hline Clinical stability on day 4 & $31(53)$ & $31(60)$ & $0(0)^{*}$ \\
\hline CRB-65 score & $1.5(0-4)$ & $1(0-4)$ & $2(1-4)$ \\
\hline \multicolumn{4}{|l|}{ CRB-65 score class } \\
\hline 0 & $13(22)$ & $13(100)$ & $0(0)$ \\
\hline 1 & $16(28)$ & $15(94)$ & $1(6)$ \\
\hline 2 & $18(31)$ & $14(78)$ & $4(22)$ \\
\hline 3 & $8(14)$ & $8(100)$ & $0(0)$ \\
\hline 4 & $3(5)$ & $2(67)$ & $1(33)$ \\
\hline PSI score & $107(12-233)$ & $103(12-184)$ & $143(134-233)^{*}$ \\
\hline \multicolumn{4}{|l|}{ PSI score class } \\
\hline 1 & $12(21)$ & $12(100)$ & $0(0)$ \\
\hline 2 & $3(5)$ & $3(100)$ & $0(0)$ \\
\hline 3 & $4(7)$ & $4(100)$ & $0(0)$ \\
\hline 4 & $20(34)$ & $20(100)$ & $0(0)$ \\
\hline 5 & 19 (33) & $13(68)$ & $6(32)$ \\
\hline $\mathrm{CIRCI}$ & $6(10)$ & $5(10)$ & $1(17)$ \\
\hline
\end{tabular}

Data are presented as $n$ (\%) or median (range), unless otherwise stated. COPD: chronic obstructive pulmonary disease; CRB-65: confusion, respiratory rate $\geqslant 30$ breaths $\cdot \mathrm{min}^{-1}$, low blood pressure (systolic value $<90 \mathrm{mmHg}$ or diastolic value $\leqslant 60 \mathrm{mmHg}$ ) and age $\geqslant 65 \mathrm{yrs}$; PSI: pneumonia severity index; CIRCl: critical illness-related corticosteroid insufficiency. ${ }^{*}: p<0.05$ for nonsurvivors versus survivors.
Levels of DHEA were elevated (median 167 (interquartile range $118-363$ ) \% of age-corrected normal values; $p<0.001$ by Wilcoxon test) in the overall cohort, whereas levels of DHEAS were not significantly different from the age- and sex-matched normal values.

Results of the measured serum parameters according to outcomes are shown in table 2. Cortisol $(p<0.001)$, ACTH $(p=0.012)$, age-corrected DHEA $(p=0.02)$ and the ratio of DHEA/DHEAS $(\mathrm{p}=0.01)$ were significantly higher in patients who did not reach clinical stability after $72 \mathrm{~h}$. Only cortisol was significantly higher in nonsurvivors $(p=0.009)$ (fig. 1).

In multivariate analysis including the three determined CAP severity scores and all measured serum parameters, only cortisol (OR 1.006 (95\% CI 1.000-1.012); p=0.03), ACTH (OR $1.346(1.018-1.780) ; \mathrm{p}=0.04)$ and the PSI score (OR 1.045 (1.013$1.078) ; p=0.005)$ independently predicted clinical instability after $72 \mathrm{~h}$, and only cortisol independently predicted mortality (OR 1.002 (1.000-1.004); $\mathrm{p}=0.04)$ and combined mortality or ICU admission (OR 1.003 (1.001-1.004); p=0.006).

The diagnostic value of serum cortisol as calculated by ROC curve analysis equalled that of the PSI score for prediction of clinical instability after $72 \mathrm{~h}$, mortality and combined ICU admission or mortality, and was superior to that of CRP, PCT and TNF- $\alpha$ (table 3). Patients with a serum cortisol concentration of $>734 \mathrm{nmol} \cdot \mathrm{L}^{-1}$ on day 1 had a significantly lower survival probability as documented by Kaplan-Meier analysis (fig. 2).

The diagnostic properties and unadjusted odds ratios of cortisol for the prediction of clinical instability after $72 \mathrm{~h}$ and mortality are shown in table 4 .

\section{DISCUSSION}

The main findings of our study are: 1) the adrenal hormones cortisol, DHEA and ACTH are related to severity and outcome of CAP; 2) CIRCI is a rare event in patients with moderate CAP; and 3) serum cortisol is superior to other serum parameters in predicting CAP outcomes and equals the prognostic accuracy of the PSI score.

Physiologically, acute stress like severe illness leads to an activation of the hypothalamic-pituitary-adrenal axis which protects the organism against excessive inflammatory responses [14]. Previous studies have shown an increase of cortisol levels that parallels the severity of infection and prognosis of patients with severe sepsis and septic shock [15]. Fewer studies have measured the adrenal hormones DHEA and DHEAS in sepsis. They are precursors of sex steroids but also demonstrate immunomodulatory effects in vitro and in vivo in the context of inflammation. Thus, they might play a regulatory role, partly as functional antagonists of the immunosuppressive effects of cortisol, during inflammatory processes [16, 17]. Therefore, a balanced response of the adrenal hormones might be crucial for an adequate adrenal response to infections. Accordingly, previous studies found an increase of DHEA in patients with septic shock (but not in trauma patients), whereas DHEAS remained stable or decreased, resulting in a dissociation of DHEA/DHEAS [1, 2]. In nonsurvivors of septic shock, despite the elevation of DHEA, a dissociation of the cortisol/DHEA 


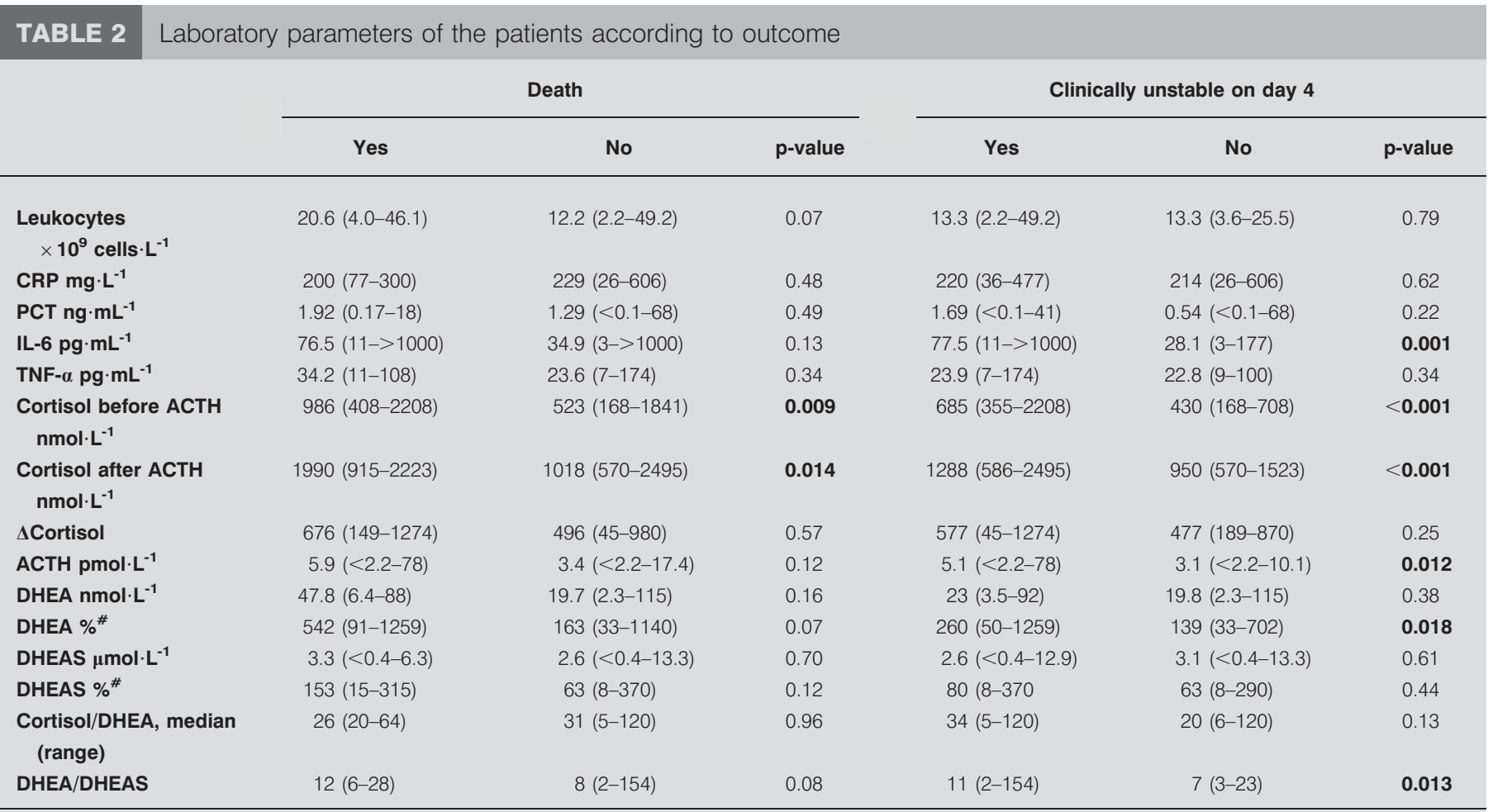

Data are presented as median (interquartile range), unless otherwise stated. Bold type indicates statistically significant p-values. CRP: C-reactive protein; PCT: procalcitonin; IL-6: interleukin-6: TNF- $\alpha$ : tumour necrosis factor- $\alpha$; ACTH: adrenocorticotrophic hormone; DHEA: dehydroepiandrosterone; DHEAS: DHEA sulphate. ${ }^{\#}$ : in $\%$ of age- (and sex-) matched normal value.

ratio was seen and a prognostic relevance of this dissociation was postulated [1,2].

Respiratory tract infections and especially CAP are a major cause of sepsis and a common precursor of the development of septic shock. To the best of our knowledge, these are the first data reporting adrenal function including levels of DHEA and DHEAS in patients with CAP hospitalised outside the ICU. As expected, cortisol levels were positively correlated with CAP severity. Moreover, we found a positive correlation with serum inflammatory parameters, especially with IL-6. Additionally, as described in septic shock patients [1], we found elevated median age-corrected levels of DHEA in the overall cohort of our patients with moderate CAP. Moreover, when compared to patients with an uncomplicated course of the disease, we found elevated age-corrected levels of DHEA and a dissociation of DHEA and DHEAS in patients who had an unfavourable outcome. Corrected DHEA values and the DHEA/ DHEAS ratio were significantly increased in patients not reaching clinical stability after $72 \mathrm{~h}$, and concordant trends were noted in patients who died. Unlike in septic shock, we did not observe a significant dissociation of cortisol and DHEA in our patients with adverse outcomes, which therefore might reflect even more advanced infection severity. However, it can be postulated that the cascade of concentration changes of adrenal hormones described in septic shock already starts in patients with moderate CAP. Whether these changes reflect adequate adrenal regulation because of more complicated disease or adrenal dysregulation resulting in a complicated infection course remains to be evaluated within larger studies.
CIRCI is frequent in patients with septic shock, occurring in up to $60 \%$, and has been repeatedly shown to be associated with a poor prognosis [3-5]. Accordingly, a study in severe CAP patients hospitalised in the ICU found a high rate of CIRCI of $41 \%$; however, CIRCI was not associated with outcome in that study [18]. In our study, only patients initially hospitalised outside the ICU were included, and a low rate of $10 \%$ of CIRCI was noted, which probably reflects the lower disease severity. Although we found a trend towards a higher probability of remaining clinically unstable after $72 \mathrm{~h}$ in patients with CIRCI on day 1, this difference was not statistically significant. According to our knowledge, only one other study examined CIRCI in patients with moderate CAP, but that Japanese study of 64 subjects, included patients on a normal ward and patients with septic shock [9]. In that study, a comparable overall rate of $14 \%$ (nine patients including three with septic shock) was found. No association with length of hospital stay and survival was described. However, clinical stability was not examined. Thus, it might be concluded that CIRCI in patients with moderate CAP can occur, but it is a rare event of uncertain significance and might not serve as a clue for an overall major treatment effect of hydrocortisone infusion in CAP patients without septic shock. However, the risk factors for and prognostic relevance of the rare event of CIRCI in CAP remains to be elucidated in larger studies.

Prognostic scores like the PSI, CURB and CRB-56 scores, are important instruments in estimating the outcome of CAP patients, and are thereby helpful for guiding treatment decisions. Additionally, several inflammatory parameters, 

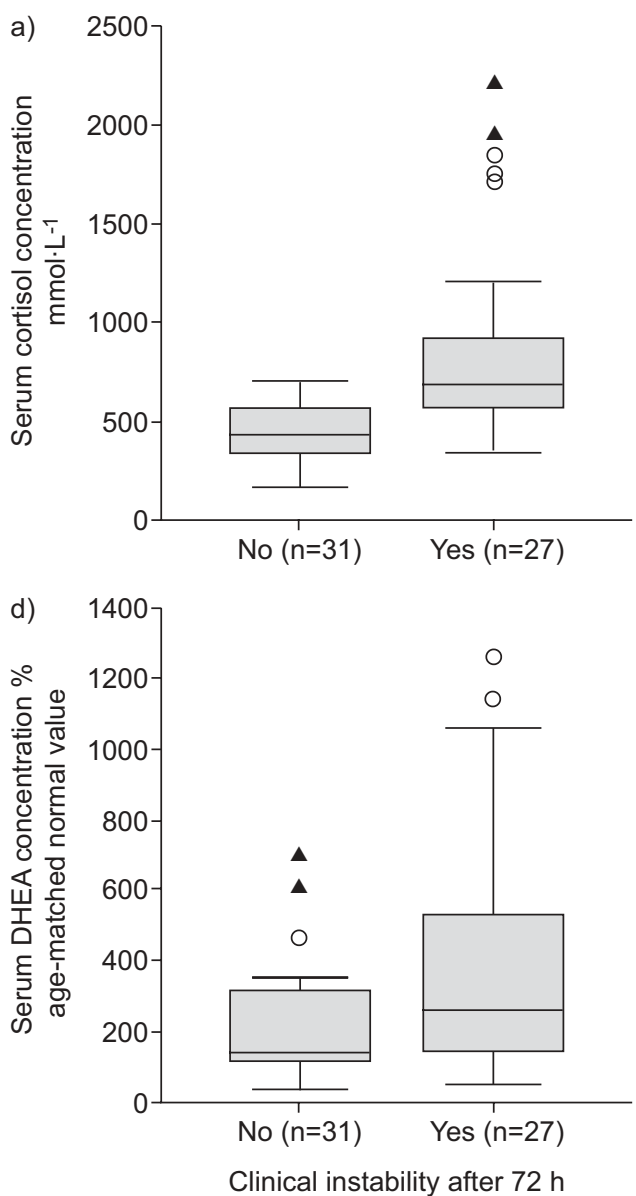

b)

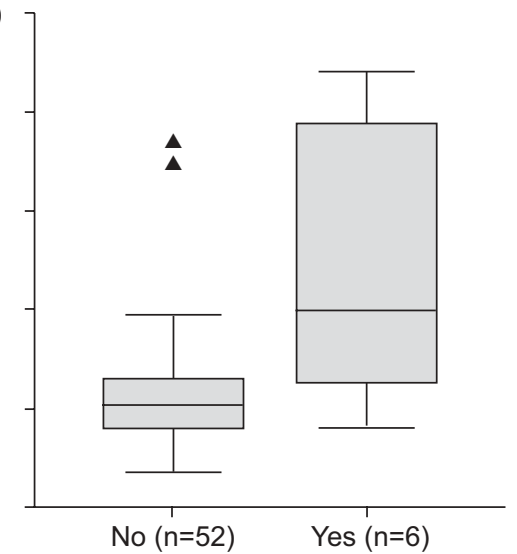

e)

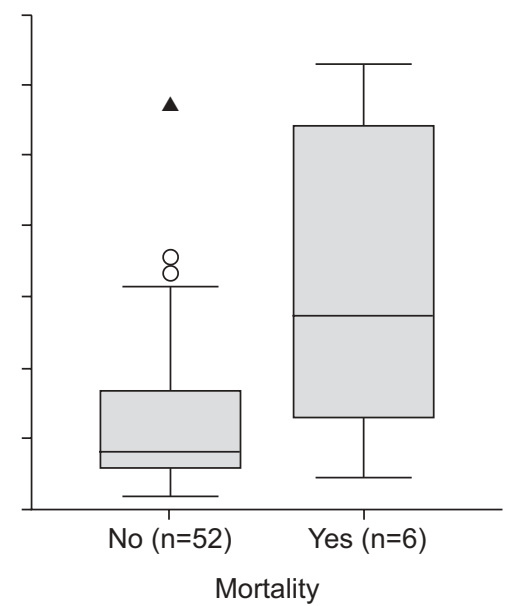

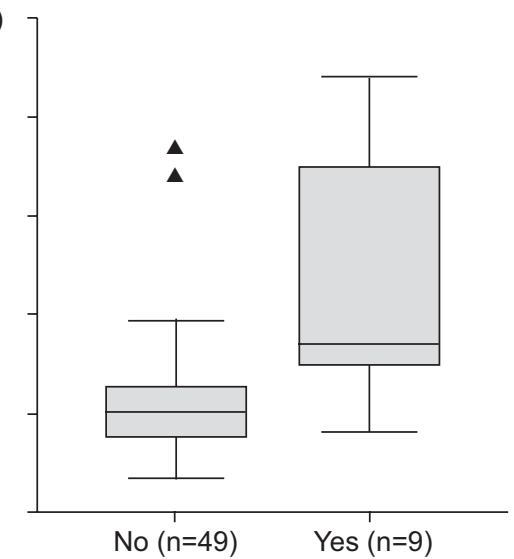

f)

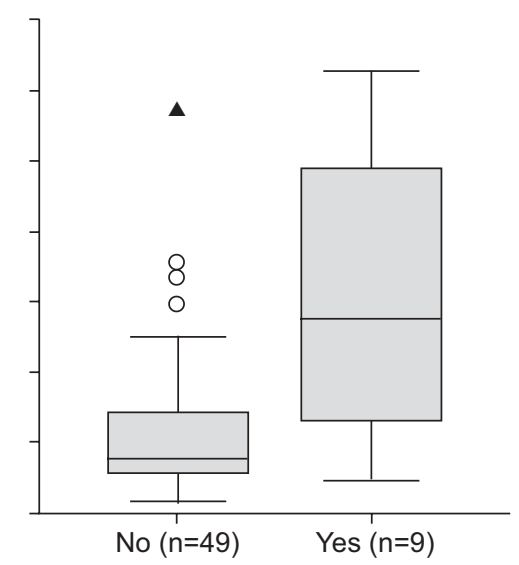

Combined ICU-admission or mortality

FIGURE 1. Box plots showing $a, b$ and $c$ ) serum concentrations of cortisol and $d$, e and $f$ ) age-corrected dehydroepiandrosterone (DHEA) according to the pre-defined outcome parameters of a and d) clinical instability after $72 \mathrm{~h}, \mathrm{~b}$ and e) mortality, and $\mathrm{c}$ and f) combined intensive care unit (ICU) admission or mortality. The box represents the 25th and 75th percentiles and the whiskers represent the 10th and 90th percentiles. -: median; $\bigcirc$ : outliers; $\mathbf{\Lambda}$ : extreme outliers.

such as CRP, PCT and IL-6, have been used for the evaluation of CAP. However, continuous need for improvement of predicting the course of CAP is necessary, and serum cortisol levels have been proposed to perform excellent prognostic properties. CHRIST-CRAIN et al. [8], in their study of 278 patients with mild to severe CAP, found that serum cortisol levels are superior to CRP and PCT in predicting mortality and have equally good prognostic accuracy as the PSI score [8]. Other

TABLE 3 Receiver operating characteristic curve statistics of cortisol, established serum parameters and established severity scores for predicting clinical instability after $72 \mathrm{~h}$, mortality, and combined intensive care unit (ICU) admission or mortality

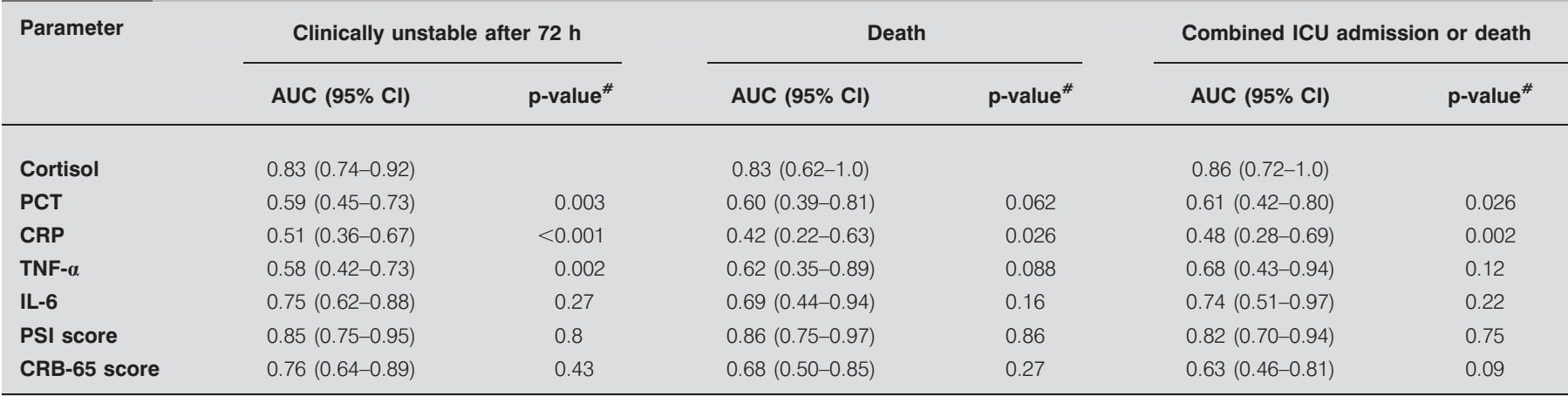

AUC: area under the curve; PCT: procalcitonin; CRP: C-reactive protein; TNF- $\alpha$ : tumour necrosis factor- $\alpha$; IL-6: interleukin-6; PSI: pneumonia severity index; CRB-65 confusion, respiratory rate $\geqslant 30$ breaths $\cdot \mathrm{min}^{-1}$, low blood pressure (systolic value $<90 \mathrm{mmHg}$ or diastolic value $\leqslant 60 \mathrm{mmHg}$ ) and age $\geqslant 65 \mathrm{yrs}$. 


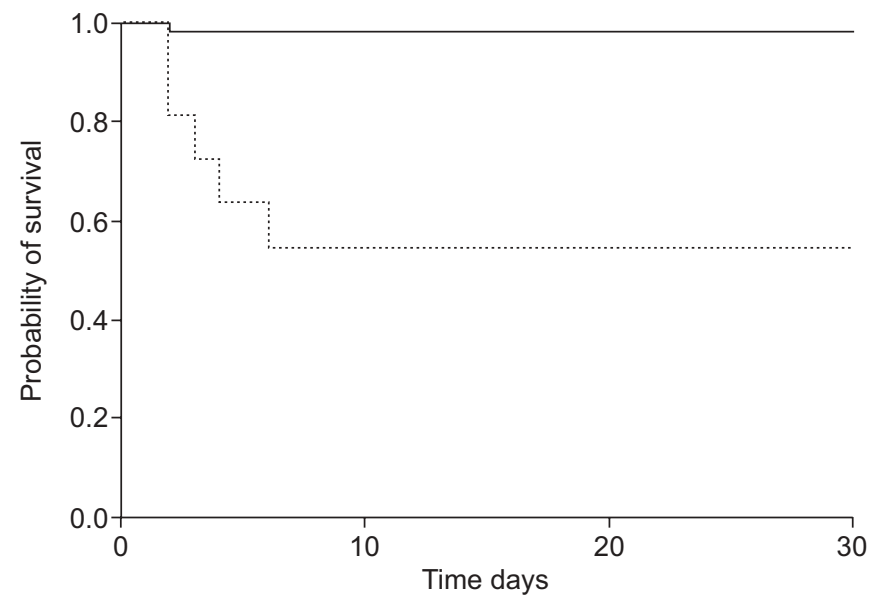

FIGURE 2. Kaplan-Meier curves showing survival rates stratified by calculated optimal serum cortisol level. —: cortisol $\leqslant 734 \mathrm{nmol} \cdot \mathrm{L}^{-1} ;$---: cortisol $>734 \mathrm{nmol} \cdot \mathrm{L}^{-1}$. $p<0.001$, as determined by log rank test.

inflammatory parameters, adrenal hormones and the CRB-65/ CURB score were not reported. GOTOH et al. [9] in their study of 64 patients with mild to severe CAP found ACTH to be a good predictor of hospital mortality (AUC 0.818) and cortisol to be a good predictor of hospital length of stay (AUC 0.818), which, on average, was relatively long at 17.7 days. Inflammatory serum parameters were not reported.

In our study, we compared the prognostic accuracy of adrenal hormones to that of the established inflammatory serum parameters CRP, PCT, IL- 6 and TNF- $\alpha$ and three established score systems for CAP. In patients with CAP hospitalised in normal wards, because of the often low mortality rate, time to clinical stability represents another validated and less confounded outcome parameter $[12,19,20]$. It is well defined by clinical parameters and reflects a very low risk of the reoccurrence of instability or death [12]. Thus, if clinical stability is reached, switch to oral antibiotics and hospital discharge can be considered [21]. We found that only ACTH, cortisol and the PSI score independently predicted clinical instability after $72 \mathrm{~h}$, and only cortisol independently predicted mortality in our cohort. As documented by ROC curve analysis, the accuracy of cortisol to predict clinical instability after $72 \mathrm{~h}$ was superior to that of other measured serum parameters and comparable to that of the difficult-to-calculate PSI score. When using a cut-off of $>571 \mathrm{mmol} \cdot \mathrm{L}^{-1}$, clinical instability after $72 \mathrm{~h}$ of treatment was predicted with a positive predictive value (PPV) of $71 \%$. A cut-off of $>734 \mathrm{mmol} \cdot \mathrm{L}^{-1}$ predicted mortality with a PPV of $45 \%$, and clinical instability after $72 \mathrm{~h}$ with a PPV of $100 \%$, and was associated with a significantly lower survival in Kaplan-Meier analysis.

PCT is a frequently used serum parameter in assessing hospitalised patients with CAP, and various studies have suggested a prognostic relevance of this parameter. However, in our cohort, as in the study by CHRIST-CRAIN et al. [8], cortisol had a comparably superior prognostic accuracy to PCT (AUC mortality for cortisol 0.827 and 0.76 , respectively; for PCT 0.60 and 0.60 , respectively). Possible concepts of the interpretation of both serum parameters thus might be that PCT predominantly has prognostic strength in defining low-risk patients by low values $[22,23]$, is an outcome parameter derived from kinetic data during treatment [24] or that it reflects a diagnostic tool able to guide decisions on antibiotic therapy [8, 25]. However, serum cortisol may have superior strength as a marker for predicting poor prognosis at hospital admission.

The main limitation of our study is the small sample size in a single-institution study, not allowing, for instance, a clear statement regarding the prognostic relevance of and possible predictors for the rare event of CIRCI in patients with CAP on a normal ward. Furthermore, we did not measure serum levels of free cortisol, which have been suggested to be more reliable, especially in the context of critical illness related hypoalbuminaemia [26]. However, although serum albumin levels in our cohort were slightly lower in patients not reaching clinical stability after $72 \mathrm{~h}$ (median levels $3.5 \mathrm{~g} \cdot \mathrm{dL}^{-1}$ versus $3.9 \mathrm{~g} \cdot \mathrm{dL}^{-1}$; $\mathrm{p}=0.004$ ), levels were not significantly different in patients who did not survive, and only three patients of our cohort had relevant hypoalbuminaemia with levels $<2.5 \mathrm{~g} \cdot \mathrm{dL}^{-1}$. Moreover, CHRIST-CRAIN et al. [8] found no difference between the prognostic values of free and total serum cortisol in a population of CAP patients comparable to ours.

In conclusion, adrenal function as measured by levels of cortisol, DHEA and ACTH on hospital admission is related to severity and outcome of CAP. CIRCI is a rare event in CAP outside the ICU. Larger studies are needed to determine its prognostic significance and risk factors. Serum cortisol has a good prognostic accuracy in predicting a high risk for clinical instability after $72 \mathrm{~h}$ and mortality, which equals that of the difficult-to-calculate PSI score. Thus, serum cortisol should be evaluated as adjunct to clinical prediction scores on larger studies.

\section{SUPPORT STATEMENT}

The study was partially funded by an award from the German Atemwegsliga.

\begin{tabular}{|c|c|c|c|c|c|c|c|}
\hline Outcome & $\begin{array}{l}\text { Cortisol cut-off } \\
\mathrm{nmol} \cdot \mathrm{L}^{-1}\end{array}$ & OR (95\% Cl) & $p$-value & $\begin{array}{l}\text { Sensitivity } \\
\%(95 \% \mathrm{Cl})\end{array}$ & $\begin{array}{l}\text { Specificity } \\
\%(95 \% \mathrm{Cl})\end{array}$ & $\begin{array}{c}\text { PPV } \\
\%(95 \% \mathrm{Cl})\end{array}$ & $\begin{array}{c}\text { NPV } \\
\%(95 \% \mathrm{Cl})\end{array}$ \\
\hline $\begin{array}{l}\text { Clinically unstable } \\
\text { after } 72 \mathrm{~h}\end{array}$ & $>571$ & $8.2(2.5-26.7)$ & $<0.001$ & 78 (58-91) & $74(55-88)$ & $71(51-87)$ & 77 (58-90) \\
\hline Death & $>734$ & $38.3(3.8-386)$ & 0.002 & 83 (36-99) & 87 (74-94) & $45(17-77)$ & $98(89-100)$ \\
\hline
\end{tabular}

PPV: positive predictive value; NPV: negative predictive value 


\section{STATEMENT OF INTEREST}

None declared.

\section{REFERENCES}

1 Arlt W, Hammer F, Sanning P, et al. Dissociation of serum dehydroepiandrosterone and dehydroepiandrosterone sulfate in septic shock. J Clin Endocrinol Metab 2006; 91: 2548-2554.

2 Marx C, Petros S, Bornstein SR, et al. Adrenocortical hormones in survivors and nonsurvivors of severe sepsis: diverse time course of dehydroepiandrosterone, dehydroepiandrosterone sulfate, and cortisol. Crit Care Med 2003; 31: 1382-1388.

3 Marik PE, Pastores SM, Annane D, et al. Recommendations for the diagnosis and management of corticosteroid insufficiency in critically ill adult patients: consensus statements from an international task force by the American College of Critical Care Medicine. Crit Care Med 2008; 36: 1937-1949.

4 Annane D, Maxime V, Ibrahim F, et al. Diagnosis of adrenal insufficiency in severe sepsis and septic shock. Am J Respir Crit Care Med 2006; 174: 1319-1326.

5 Lipiner-Friedman D, Sprung CL, Laterre PF, et al. Adrenal function in sepsis: the retrospective Corticus cohort study. Crit Care Med 2007; 35: 1012-1018.

6 Sprung CL, Annane D, Keh D, et al. Hydrocortisone therapy for patients with septic shock. N Engl J Med 2008; 358: 111-124.

7 Confalonieri M, Urbino R, Potena A, et al. Hydrocortisone infusion for severe community-acquired pneumonia: a preliminary randomized study. Am J Respir Crit Care Med 2005; 171: 242-248.

8 Christ-Crain M, Stolz D, Jutla S, et al. Free and total cortisol levels as predictors of severity and outcome in community-acquired pneumonia. Am J Respir Crit Care Med 2007; 176: 913-920.

9 Gotoh S, Nishimura N, Takahashi O, et al. Adrenal function in patients with community-acquired pneumonia. Eur Respir J 2008; 31: 1268-1273.

10 Labrie F, Belanger A, Cusan L, et al. Marked decline in serum concentrations of adrenal C19 sex steroid precursors and conjugated androgen metabolites during aging. J Clin Endocrinol Metab 1997; 82: 2396-2402.

11 Hoffken G, Lorenz J, Kern W, et al. [S3-guideline on ambulant acquired pneumonia and deep airway infections.] Pneumologie 2005; 59: 612-664.

12 Halm EA, Fine MJ, Marrie TJ, et al. Time to clinical stability in patients hospitalized with community-acquired pneumonia: implications for practice guidelines. JAMA 1998; 279: 1452-1457.
13 Hanley JA, McNeil BJ. A method of comparing the areas under receiver operating characteristic curves derived from the same cases. Radiology 1983; 148: 839-843.

14 Cooper MS, Stewart PM. Corticosteroid insufficiency in acutely ill patients. N Engl J Med 2003; 348: 727-734.

15 Arafah BM. Hypothalamic pituitary adrenal function during critical illness: limitations of current assessment methods. J Clin Endocrinol Metab 2006; 91: 3725-3745.

16 Loria RM. $\beta$-androstenes and resistance to viral and bacterial infections. Neuroimmunomodulation 2009; 16: 88-95.

17 Oberbeck R. Therapeutic implications of immune-endocrine interactions in the critically ill patients. Curr Drug Targets Immune Endocr Metabol Disord 2004; 4: 129-139.

18 Salluh JI, Bozza FA, Soares M, et al. Adrenal response in severe community-acquired pneumonia: impact on outcomes and disease severity. Chest 2008; 134: 947-954.

19 Ramirez JA, Srinath L, Ahkee S, et al. Early switch from intravenous to oral cephalosporins in the treatment of hospitalized patients with community-acquired pneumonia. Arch Intern Med 1995; 155: 1273-1276.

20 Menendez R, Torres A, Rodriguez de Castro F, et al. Reaching stability in community-acquired pneumonia: the effects of the severity of disease, treatment, and the characteristics of patients. Clin Infect Dis 2004; 39: 1783-1790.

21 Mandell LA, Wunderink RG, Anzueto A, et al. Infectious Diseases Society of America/American Thoracic Society consensus guidelines on the management of community-acquired pneumonia in adults. Clin Infect Dis 2007; 44: S27-S72.

22 Kruger S, Ewig S, Marre R, et al. Procalcitonin predicts patients at low risk of death from community-acquired pneumonia across all CRB-65 classes. Eur Respir J 2008; 31: 349-355.

23 Huang DT, Angus DC, Kellum JA, et al. Midregional proadrenomedullin as a prognostic tool in community-acquired pneumonia. Chest 2009; 136: 823-831.

24 Charles PE, Tinel C, Barbar S, et al. Procalcitonin kinetics within the first days of sepsis: relationship with the appropriateness of antibiotic therapy and the outcome. Crit Care 2009; 13: R38.

25 Christ-Crain M, Stolz D, Bingisser R, et al. Procalcitonin guidance of antibiotic therapy in community-acquired pneumonia: a randomized trial. Am J Respir Crit Care Med 2006; 174: 84-93.

26 Hamrahian AH, Oseni TS, Arafah BM. Measurements of serum free cortisol in critically ill patients. New Engl J Med 2004; 350: 1629-1638. 